\title{
ASCA Observations of LINERs
}

\author{
Y. Terashima, ${ }^{1}$ H. Kunieda, ${ }^{1}$ N. Iyomoto,${ }^{2}$ K. Makishima,${ }^{2}$ and P. J. \\ Serlemitsos $^{3}$ \\ ${ }^{1}$ Department of Physics, Nagoya University, Chikusa-ku, Nagoya, Japan \\ ${ }^{2}$ Department of Physics, University of Tokyo, Bunkyo-ku, Tokyo, Japan \\ ${ }^{3}$ NASA/Goddard Space Flight Center, Greenbelt, USA
}

\section{Introduction}

ASCA observations have revealed the presence of low-luminosity AGN in 10 LINERs as a hard point sources at the nucleus. The X-ray continuum shapes (photon indices $\Gamma \approx 1.8$ ) are very similar to those of Seyfert galaxies (Makishima et al. 1997, Serlemitsos et al. 1996). An iron emission line is observed from heavily absorbed low-luminosity AGNs (Makishima et al. 1997), while M 81 is the only object among those with small intrinsic absorption from which an iron line detected (Ishisaki et al. 1996) on account of limited photon statistics for other objects.

\section{Composite Spectrum of LINERs}

We summed up the $A S C A$ spectra of 5 LINERs which host low-luminosity AGN of low intrinsic absorption to search for an iron emission line. We used 7 observations of 5 objects (NGC 1097, NGC 3310, NGC 3998, NGC 4450, and NGC 4594) to make a composite spectrum. All the objects have very similar $\mathrm{X}$-ray characteristics (spectral slope, intrinsic absorption, no short time-scale variability; Iyomoto et al. 1996, Serlemitsos et al. 1996).

We fitted this spectrum with a power-law model in the $2-10 \mathrm{keV}$ band, since most of the $A S C A$ spectra of LINERs indicate thermal emission from a hot gas of $k T \approx 0.5 \mathrm{keV}$, and such a component is negligible above $2 \mathrm{keV}$.

The best-fit photon index is $\Gamma=1.79 \pm 0.04$. Only an upper limit of equivalent width (EW) $140 \mathrm{eV}$ was obtained for an additional narrow Gaussian feature at $6.4 \mathrm{keV}$ or $6.7 \mathrm{keV}$, which corresponds to nearly neutral and He-like iron, respectively ( $90 \%$ confidence limit for one parameter of interest).

From the prototypical LINER M 81, a broad iron emission line (equivalent width $\sim 300 \mathrm{eV}$, Gaussian-line width $\sigma \approx 0.2-0.3 \mathrm{keV}$ ) is clearly detected at $\sim 6.7 \mathrm{keV}$ (Serlemitsos et al. 1996, Ishisaki et al. 1996), which is compatible with an accretion-disk origin (Serlemitsos et al. 1996) as observed in Seyfert 1 galaxies (e.g., Nandra et al. 1996). However, these parameters are inconsistent with the average LINER spectrum.

If an optically thick accretion disk is present in LINERs as in Seyfert galaxies, in spite of very low Eddington ratio (e.g., $\sim 7 \times 10^{-6}$ for NGC 4258; Makishima et al. 1994, Miyoshi et al. 1995), the weakness of iron lines in LINERs 

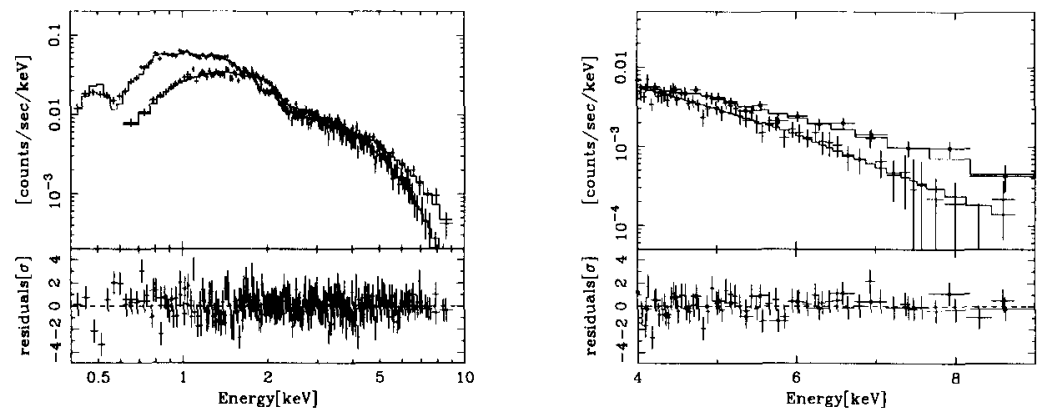

Figure 1. The $A S C A$ data and the best-fit power-law plus thin thermal plasma model.

would be explained by inclination and/or ionization-state dependence of the EW. When the iron line originates from a cold disk, the EW decreases as the inclination becomes large (George \& Fabian 1991). Furthermore, the iron line profile from a relativistic disk with higher inclination becomes very broad (Fabian et al. 1989). Thus iron lines from highly inclined disks are hard to detect.

Effective fluorescence yield of iron becomes small for Fe XVII -FeXXIII due to the resonance trapping effect (e.g., Życki \& Czerny 1994, Ross \& Fabian 1993). When the ionization state of the iron lies in this range, iron emission is suppressed. In this case, the strong iron line in M 81 could be interpreted as arising in more highly ionized species which have larger effective fluorescence yields.

Alternatively it is also possible that LINERs have no standard accretion disk (Mushotzky 1993). The absence of the reprocessor around the nucleus easily explains lack of the iron line. In this case, however, the origin of the iron line in M 81 may be a problem.

\section{References}

Fabian, A. C., et al. 1989, MNRAS, 238, 729.

George, I. M., \& Fabian, A. C. 1991, MNRAS, 249, 352.

Ishisaki, Y., et al. 1996, PASJ, 48, 237.

Iyomoto, N., et al. 1996, PASJ, 48, 231.

Makishima, K., et al. 1994, PASJ, 46, L77.

Makishima, K., et al. 1997, this volume.

Miyoshi, M., et al. 1995, Nature, 373, 127.

Mushotzky, R.F. 1993, in The Nearest Active Galaxies, eds. J. Beckman, L. Colina, \& H. Netzer.

Nandra, K., et al. 1996, ApJ, in press.

Serlemitsos, P.J., Ptak, A.F., \& Yaqoob, T. 1996, in The Physics of LINERs. Życki, P. T., \& Czerny, B. 1994, MNRAS, 266, 653. 